\title{
How to Create a National Quality Framework for Palliative Care? Experience from the Netherlands
}

Boddaert M.S.A. ${ }^{1,2}$, Dijxhoorn A.F.Q. ${ }^{12}$, Douma J. ${ }^{1,2,3}$, Larkin P.J. ${ }^{4,5}$, Huijgens P.C. ${ }^{1}$, van der Rijt C.C.D. ${ }^{3,6}$, Vissers K.C.P. ${ }^{3,7}$

'Netherlands Comprehensive Cancer Organisation (IKNL), Utrecht, Netherlands; ${ }^{2}$ Association for Palliative Care in the Netherlands (PZNL), Utrecht, Netherlands; ${ }^{3}$ Palliactief Amsterdam, Netherlands: ${ }^{4}$ Université de Lausanne Lausanne, Switzerland; ${ }^{5}$ Centre hospitalier universitaire vaudois, Lausanne Switzerland: 6 Erasmus University Medical Center, Oncology, Rotterdam, Netherlands; 7 Radboud University Medical Center, Department of Anaesthesiology, Pain and Palliative Medicine, Nijmegen, Netherlands

\section{BACKGROUND}

In the Netherlands, palliative care (PC) is part of a national program for the improvement of quality of life and care for patients with life-threatening conditions. Hence, the Ministry of Health encourages healthcare professionals and affiliated organizations to provide primary PC, supported by palliative care specialists, when required. Here we present the process of development of a national quality framework for PC, the requisite consensus-building and main topics of content.

\section{AIM}

To create a broadly supported consensus based quality framework for PC for patients with a life-threatening condition or frailty and their families, based on their values, wishes and needs.

\section{METHOD}

Analogous to Dutch national guideline procedures, a working group comprising mandated representatives of patients and their families, scientific societies of various healthcare providers and health insurers formulated the key question: "What do we agree the criteria for quality of palliative care in the Netherlands should be".
Constraint analyses for PC in Dutch primary and secondary care settings and a literature search were carried out. Four international and two national core documents were used in a Delphi method to build the framework with the working group and stakeholder organizations were kept informed at management level. The whole process took one year of preparing and building relationships and two years of convening, discussing content and consulting peers.

\section{RESULTS}

A quality framework, including a glossary of terms endorsed by organizations representing patients and their families, general practitioners, elderly care physicians, medical specialists, nurses, social workers, psychologists, spiritual caregivers and health insurers was launched. In addition, a summary for patients and families and a list of recommended measurement instruments were developed.

\section{CONCLUSION}

A continuous whole-of-sector approach is a feasible way to develop a national consensus- and literature based quality framework.
INTERNATIONAL LITERATURE INVENTORY This decision tree reflects
the search process of the international inventory for determining
the core documents. A similar inventory was made for national the core docure, adding to a total of six core documents.

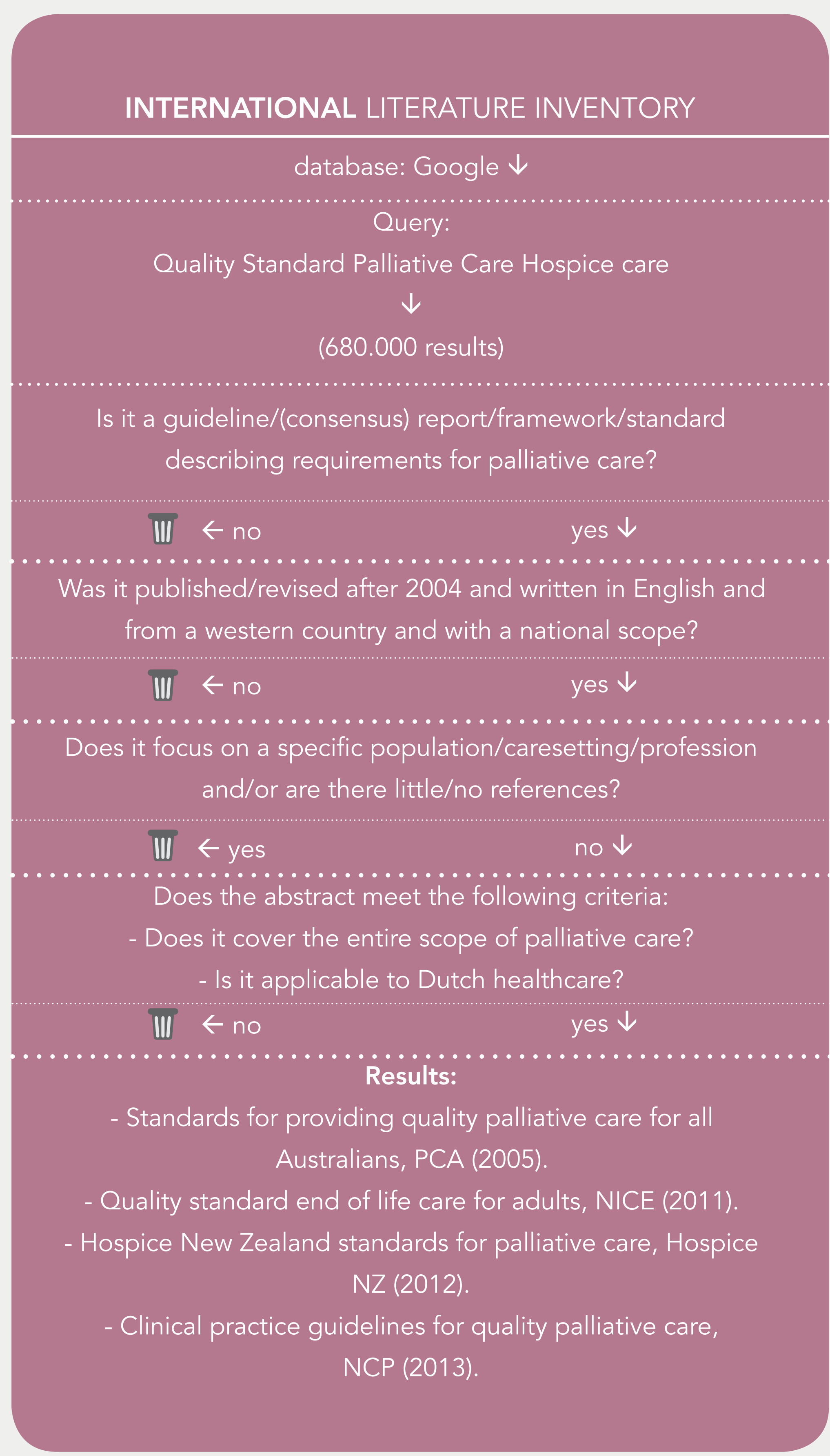

TEN DOMAINS OF THE NETHERLANDS QUALITY FRAMEWORK FOR PALLIATIVE CARE The quality framework consists of ten domains. An introduction provides a broad outline of each domain and standards supported by criteria indicate best practice.
The Netherlands Quality Framework for Palliative Care has been endorsed by the following organisations:

DOMAIN 1: Core values and principles

\section{DOIVAN 2. Siructure and process}

DOMAIN 3: Physical dimension DOMAIN 4: Psychological dimension DOMAIN 5: Social dimension DOMAIN 6: Spiritual dimension DOMAIN 7: End-of-life care DOMAIN 8: Loss and bereavement DOMAIN 9: Cultural aspects DOMAIN 10: Ethical and legal aspects

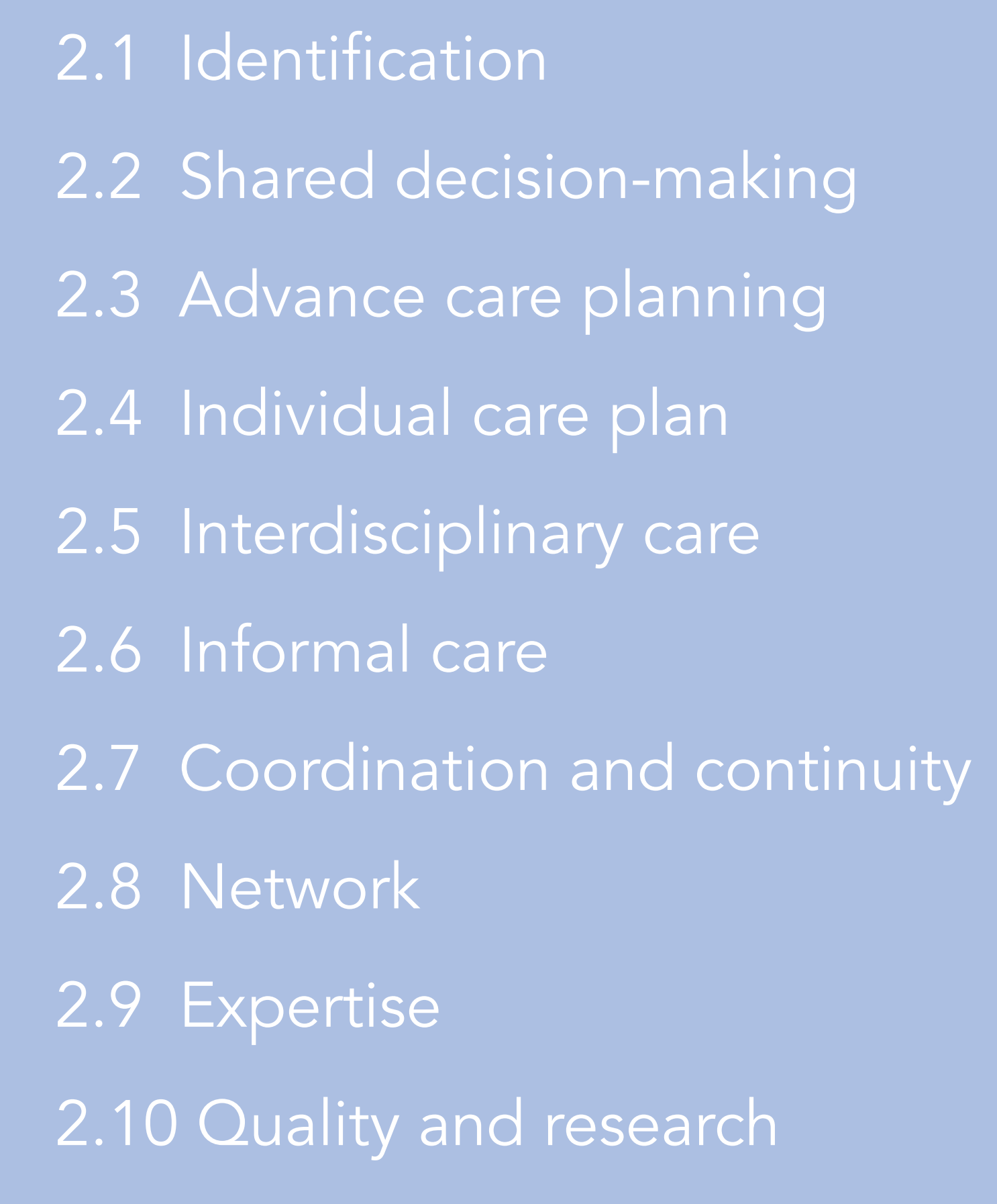
Nederland

\section{verenso}
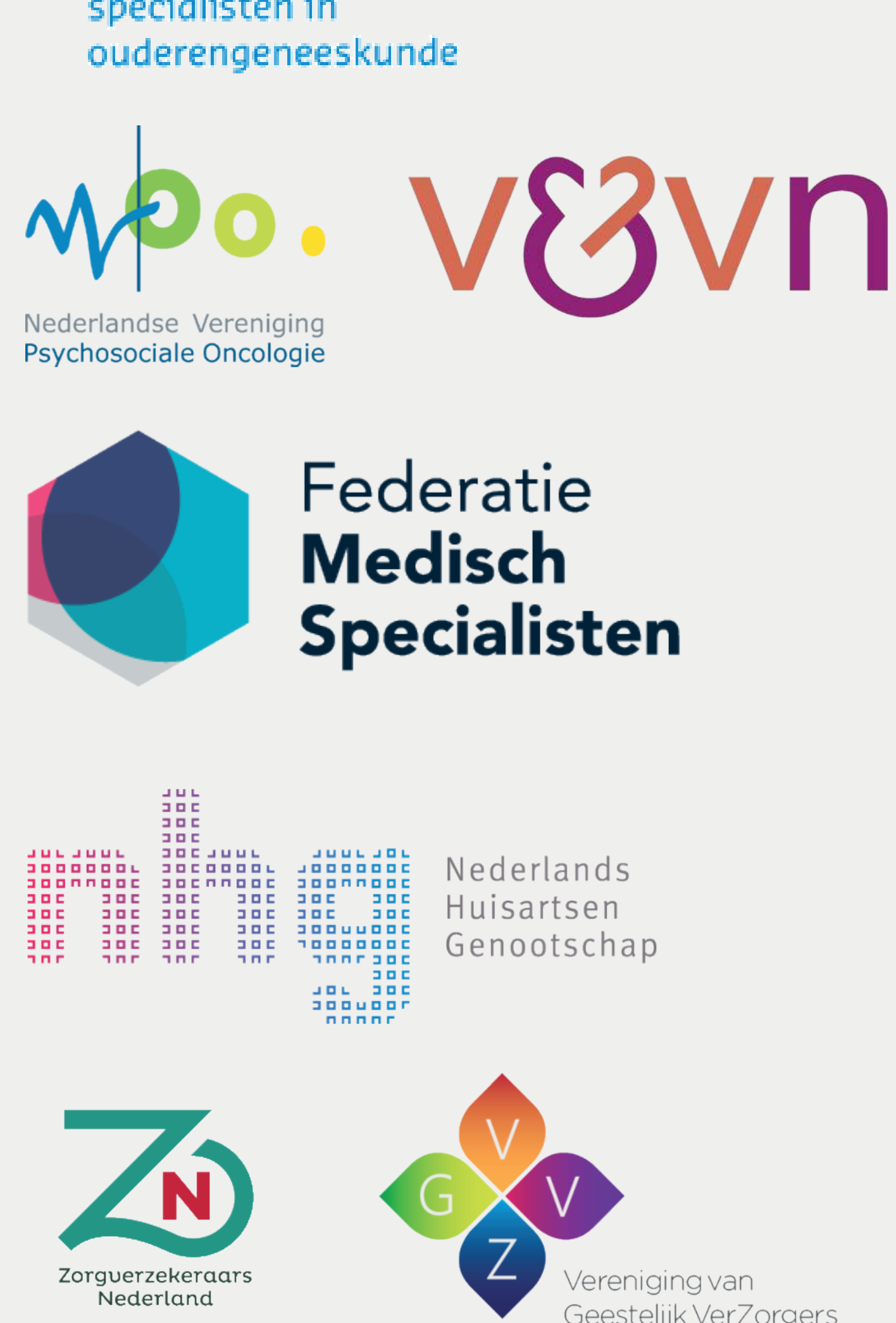

Q Patiëntenfederatie

Development of the quality framework was initiated by Palliactief and IKNL (member of cooperation PZNL) 